\title{
5.03
}

\section{Levantamento da capacidade de avaliação dos projetos de estabelecimentos assistenciais de saúde pelas vigilâncias sanitárias}

Detection of capacity of evaluating health facilities projects by health surveillance teams.

\section{Adjane Balbino de Amorim Rodrigues}

Arquiteta e Urbanista, Mestre em Arquitetura. Especialista em Regulação e Vigilância Sanitária, Agência Nacional de Vigilância Sanitária. Brasília, Brasil.

\section{Letícia Lopes Quirino Pantoja}

Odontóloga. Especialista em Regulação e Vigilância Sanitária, Agência Nacional de Vigilância Sanitária. Brasília, Brasil.

Resumo: Em outubro de 2011, foi publicada a Resolução Anvisa n. 51, que dispõe sobre requisitos mínimos para a análise, avaliação e aprovação dos projetos físicos de estabelecimentos de saúde pelo Sistema Nacional de Vigilância Sanitária (SNVS). A avaliação do projeto arquitetônico de um estabelecimento assistencial de saúde (EAS) é o primeiro ato de vigilância sanitária. Para a avaliação de um projeto de infraestrutura é necessário compor uma equipe multidisciplinar, que deva possuir entre os profissionais um legalmente habilitado pelos conselhos de arquitetura ou engenharia. Para conhecer a força de trabalho nas vigilâncias sanitárias do País que são as responsáveis pela aprovação dos projetos de infraestrutura, a Gerência de Regulamentação e Controle Sanitário (GRECS) da Anvisa propôs um censo sobre o número de profissionais legalmente habilitados para proceder com a análise de projetos. Esse levantamento foi realizado por meio do FormSus. O resultado pode ser constatado por região doPaís no artigo: avaliação dos projetos de estabelecimentos assistenciais de saúde pelas vigilâncias sanitárias. O levantamento realizado da capacidade do SNVS para a avaliação de projetos arquitetônicos constituiu-se em subsídio para as ações de planejamento da Anvisa, na sua função de coordenação do SNVS,com vistas à redução dos riscos à saúde da população. Este artigo descreve, além da metodologia do levantamento, um exemplo da aplicação do seu resultado no planejamento das ações da Anvisa na implantação das políticas públicas do Ministério da Saúde relacionadas a ampliação do acesso aos serviços de saúde.

Palavras chave: avaliação projetos arquitetônicos; vigilância sanitária, planejamento.

Key-words: architectural projects evaluation; sanitary surveillance, planning. 


\section{Introdução}

Em outubro de 2011, foi publicada pela Anvisa a Resolução da Diretoria Colegiada $(\mathrm{RDC})$ n. 51, que dispõe sobre requisitos mínimos para análise, avaliação e aprovação dos projetos físicos de estabelecimentos de saúde pelo Sistema Nacional de Vigilância Sanitária (SNVS). Essa resolução revogou a RDC/Anvisa n. 189, de 2003, que discorria sobre o mesmo tema.

Desde a reforma administrativa do Estado Brasileiro, iniciada em 1995, o modelo de gestão das ações governamentais baseou-se na integração dos instrumentos de planejamento, orçamento e gestão (Nader, 2005). Nesse sentido, o levantamento da capacidade do SNVS para a avaliação de projetos arquitetônicos constitui subsídio importante para as ações de planejamento da Anvisa, em virtude de sua função de coordenação do SNVS, com vistas à redução dos riscos à saúde da população (RDC 51/2011).

A avaliação do projeto arquitetônico de um estabelecimento assistencial de saúde (EAS) é o primeiro ato de vigilância sanitária. São alcançados por aquela resolução todos os EAS públicos, privados, civis e militares, inclusive os que exercem ensino e pesquisa. As obras que devem ser avaliadas pelo SNVS abrangem as novas, as áreas a serem ampliadas, as reformas e também as edificações que anteriormente não eram destinadas à saúde.

Um projeto que considere as normas vigentes de controle sanitário caracterizase como um projeto seguro, que não acarreta riscos à saúde e confere segurança à população (RDC 51/2011). Decorre dessa premissa o fato de que, para a avaliação de um projeto de infraestrutura, é necessária a existência de uma equipe multidisciplinar, que deva possuir, entre os profissionais que a compõem, ao menos um legalmente habilitado pelos conselhos de classe.

A avaliação dos projetos físicos de infraestrutura pelos agentes da Vigilância Sanitária local é uma ferramenta que o órgão de fiscalização possui para induzir que os EAS sejam edificados de acordo com as normas sanitárias, que atendam às demandas de saúde da região em que serão implantados, além de colaborar com a segurança do paciente e dos profissionais de saúde que irão compor o staff do estabelecimento. 
Algumas das inovações propostas pela nova resolução foram:

- possibilitar às vigilâncias sanitárias a consultoria específica, quando o objeto da análise requerer conhecimento complementar da equipe multidisciplinar;

- definição, pela Vigilância Sanitária local, dos prazos para a entrega dos pareceres, resguardado o direito de obtenção de informações por parte do proponente; e

- introdução do prazo de validade do projeto aprovado e do parecer técnico.

Após a publicação da norma, foi realizada uma videoconferência para apresentação das novas orientações à comunidade sanitária.

Segundo a teoria de administração pública, toda ação deve ser precedida de planejamento baseado no conhecimento da realidade (Pereira, 1996). Portanto, no que se refere às ações para indução da segurança sanitária dos EAS, é necessário o conhecimento da força de trabalho das Vigilâncias Sanitárias do País, instâncias que são responsáveis pela análise e aprovação dos projetos físicos de infraestrutura.

\section{Materiais e métodos}

A Gerência de Regulamentação e Controle Sanitário (GRECS) da Anvisa propôs um censo dos profissionais legalmente habilitados para proceder a análise de projetos de infraestrutura nos estados e municípios brasileiros. Esse levantamento foi realizado por meio de um formulário eletrônico, elaborado com a ferramenta FormSUS, e divulgado junto às Vigilâncias Sanitárias Estaduais via Rede Ágorass, além de outros meios de comunicação com o SNVS. A RedeÁgorass, criada em 2011, é um espaço para comunicação rápida entre a Anvisa e as Vigilâncias Sanitárias de estados e capitais, onde são tratadas questões relacionadas às práticas de regulação e fiscalização sanitárias de serviços de saúde, especificamente.

O levantamento realizado focou em quatro questões principais relacionadas à avaliação dos projetos arquitetônicos de EAS:

- descentralização da ação de avaliação dos projetos físicos; 
- utilização da recente resolução para avaliação dos projetos físicos;

- número de profissionais habilitados para a avaliação dos projetos físicos; e

- vínculo dos profissionais habilitados com a administração pública local.

\section{Resultado e discussão}

O resultado do levantamento da capacidade de avaliação dos projetos de EAS pelas Vigilâncias Sanitárias pode ser constatado, por região, conforme as figuras abaixo.

Dos 23 estados que responderam ao formulário, verificou-se que existe descentralização das ações de avaliação de projetos arquitetônicos para alguns municípios, além da própria capital, em nove estados. 


\section{Profissionais habilitados para a análise dos Projetos Básicos de Arquitetura pelas Vigilâncias Sanitárias (em julho/2012)}

Figura 1. Região Nordeste

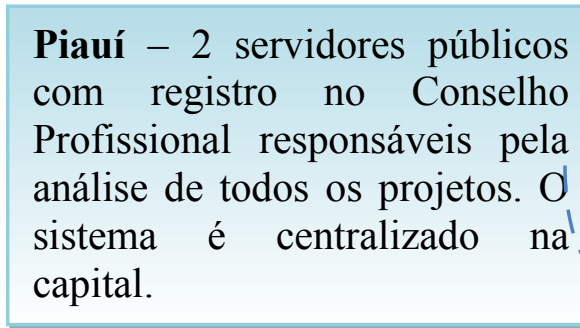

Bahia - Apenas 1 profissional contratado com registro no Conselho Profissional analisa todos os projetos. Não há servidor público no quadro. O sistema é centralizado na capital.

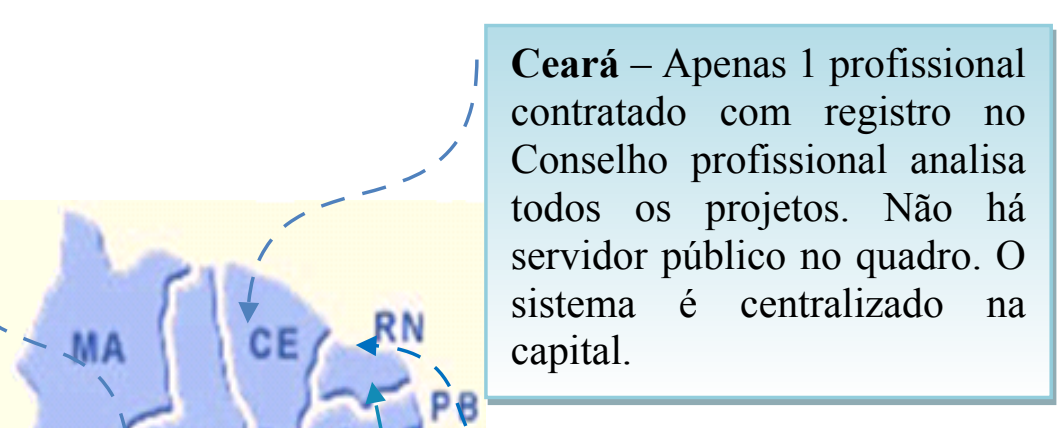

Rio Grande do Norte - 4 servidores públicos e 1 profissional contratado com registro no Conselho Profissional analisam os projetos. O sistema é centralizado na capital.

Alagoas -1 servidor público com
registro no Conselho Profissional
analisa os projetos. O sistema é
centralizado na capital.

* Os estados do Maranhão e Sergipe não responderam ao formulário.

* O estado de Pernambuco respondeu a pesquisa por telefone. 


\section{Figura 2. Região Centro Norte}

\begin{tabular}{|c|c|c|}
\hline $\begin{array}{l}\text { Amazonas }-3 \text { servidores } \\
\text { públicos e } 3 \text { profissionais } \\
\text { contratados com registro no } \\
\text { Conselho } \\
\text { analisam or profissiona } \\
\text { sistema é centralizado na } \\
\text { capital. }\end{array}$ & $\begin{array}{l}\text { com registro no Conselho } \\
\text { Profissional analisa os projetos. } \\
\text { O sistema é centralizado na } \\
\text { capital. }\end{array}$ & $\begin{array}{l}\text { Tocantins - } 1 \text { servidor público } \\
\text { e } 1 \text { profissional contratado } \\
\text { com registro no Conselho } \\
\text { Profissional analisam os } \\
\text { projẹtos. O sistema é } \\
\text { centralizado na capital. }\end{array}$ \\
\hline $\begin{array}{l}\text { Acre - } 3 \text { servidores } \\
\text { públicos e } 3 \text { profissionais } \\
\text { contratados com registro } \\
\text { no Conselho Profissional } \\
\text { analisam os projetos. O } \\
\text { sistema é centralizado na } \\
\text { capital. }\end{array}$ & AN & $\begin{array}{l}\text { Rondônia - } 5 \text { servidores } \\
\text { públicos com registro no } \\
\text { Conselho Profissional } \\
\text { analisam os projetos. O } \\
\text { sistema é centralizado na } \\
\text { capital. }\end{array}$ \\
\hline
\end{tabular}

* Os estados do Pará e Amapá não responderam ao formulário.

Figura 3. Região Centro-Oeste

Mato Grosso - 2 servidores
públicos e 1 profissional
contratado com registro no
Conselho rassional
analisam os projetos. O
sistema é centralizado na
capital.

Mato Grosso do Sul - 1 servidor público com registro no Conselho Profissional analisa os projetos. O sistema é centralizado na capital.
Goiás - 2 servidores públicos ‘com registro no Conselho /Profissional analisam os projetos. O sistema é centralizado na capital. 
Figura 4. Região Sudeste

Minas Gerais - 15 servidores
públicos e 3 profissionais
contratados com registro no
Conselho r
analisam orofissional
$\begin{aligned} & \text { sistema é centralizado na } \\ & \text { capital. }\end{aligned}$

São Paulo - 65 servidores públicos e 8 profissionais contratados com registro no conselho profissional analisam os projetos. $\mathrm{O}$ sistema é descentralizado nas cidades de São Paulo, Franco da Rocha, Santo André, Mogi das Cruzes, Osasco, Araçatuba, Araraquara, Assis, Barretos, Marília, Bauru, Botucatu, Campinas, Franca, Piracicaba, Presidente Prudente, Presidente Venceslau, Registro, Ribeirão Preto, São José do Rio Preto, Baixada Santista, São João da Boa Vista, São José dos Campos, Caraguatatuba, Jales, Sorocaba, Itapeva, Taubaté, Sorocaba e Itapetininga.
Espírito Santo - 1 servidor público e 1 profissional contratado com registro no ConselhoProfissional analisam os projetos. O sistema é descentralizado nas cidades de Vitória e Cachoeiro.

\section{Rio de Janeiro - 08 servidores públicos com registro no Conselho Profissional analisam os projetos. O sistema é centralizado na capital.}

\section{Figura 5. Região Sul}

Paraná - 13 servidores públicos
com registro no conselho
profissional analisam os projetos. O
sistema é descentralizado nas
cidades de Curitiba, Paranaguá,
Ponta Grossa, Guarapuava, União
da Vitória, Francisco Beltrão, Foz
do Iguaçú, Cascavel, Campo
Mourão, Umuarama, Cianorte,
Paranavaí, Maringá, Apucarana,
Londrina, Cornélio Procópio,
Jacarezinho, Toledo, Telêmaco
Borba, Ivaiporã, Marialva, São
Jorge do Ivaí, Assaí, São Jorge do
Oeste, Campo Largo, Colombo,
Pinhais, São José dos Pinhais,
Ibiporã, Centenário do Sul,
Araucária, Morretes.

Santa Catarina - 4 servidores públicos com registro no Conselho Profissional analisam os projetos. O sistema é descentralizado nas cidades de Florianópolis, Caçador, Canoinhas, Joinville e Mafra. 
A enquete questionou ainda sobre a aplicação da RDC/Anvisa n. 51 de 2011 (que substituiu a RDC/Anvisa n. 189/2003) pelos responsáveis pela análise dos projetos de infraestrutura nos estados e municípios (Gráfico 1).

Gráfico 1. Utilização da RDC/Anvisa n.51 de 2011

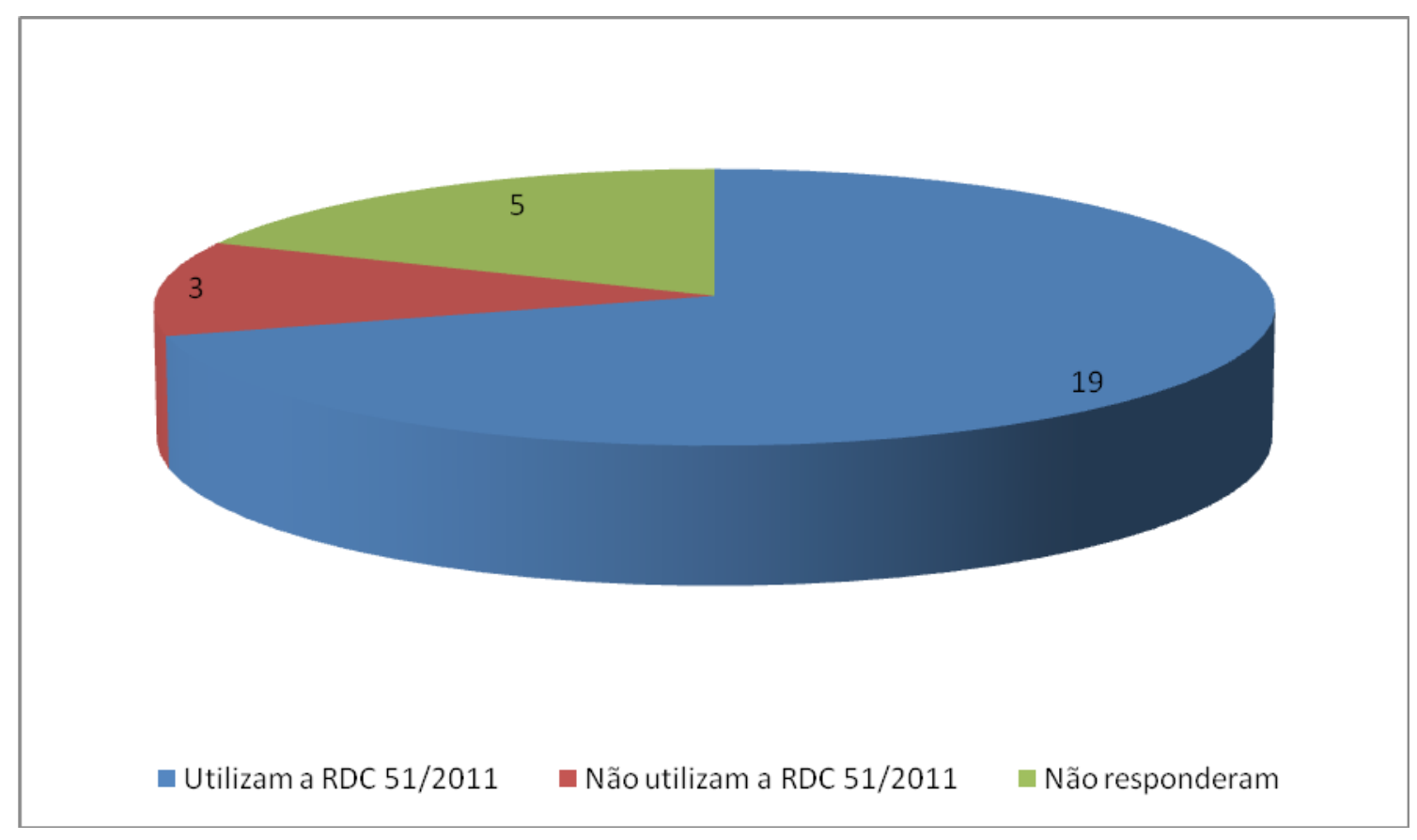

Fonte: Respostas ao questionário FormSus. As autoras

Por meio da análise dos dados presentes no Gráfico 1, verificou-se que, na época em que o levantamento foi realizado, a RDC/Anvisa n. 51, de 2011, já havia sido incorporada pela a grande maioria das Vigilâncias Sanitárias dos estados e municípios.

O levantamento do número de profissionais habilitados e a localização deles pelo País permitiu o diagnóstico da situação atual das equipes de análise de projetos de arquitetura pelas Vigilâncias Sanitárias, conforme demonstrado no Gráfico 2. 
Gráfico 2. Número de profissionais habilitados por região.

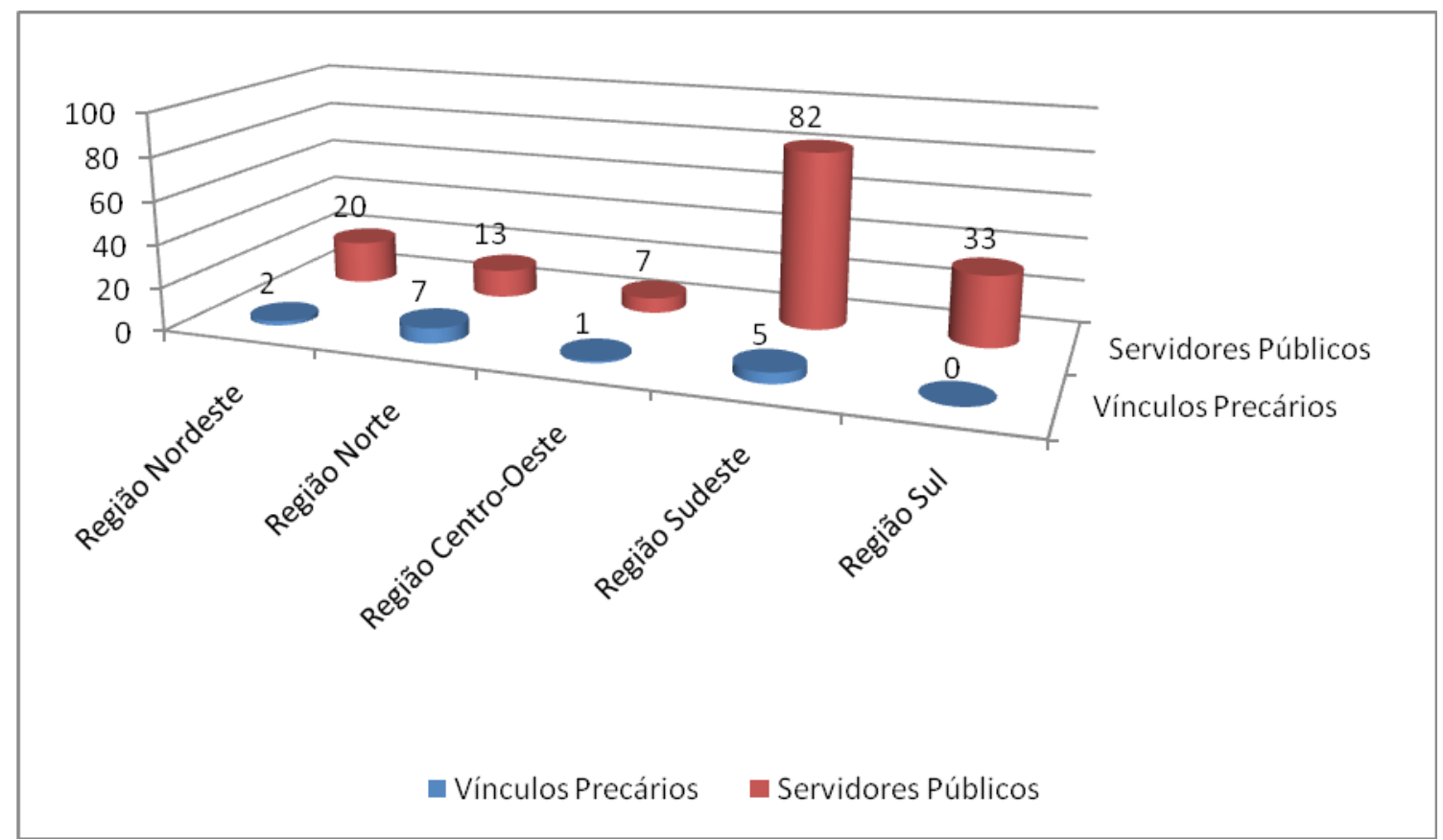

Fonte:Respostas ao questionário FormSus. As autoras

* Os estados do Maranhão, Sergipe, Pará e Amapá não responderam o formulário enviado.

* O estado de Pernambuco respondeu a pesquisa por telefone.

Com relação ao número de profissionais habilitados e ao vínculo desses com a administração pública local, observou-se que as regiões Sudeste e Sul concentram a maioria dos servidores públicos, sendo que na região Sul não houve relato da presença de profissionais com vínculo profissional precário, ou seja, sem estabilidade.

Em relação ao número de profissionais e sua distribuição entre estados e municípios em que há descentralização da atividade, não foi possível identificar, por meio do formulário aplicado, a quantidade de profissionais lotados no próprio estado ou distribuídos nos municípios. Existe ainda a possibilidade de que alguns estados que informaram atividade descentralizada não terem informado o número de profissionais lotados nos municípios.

As perguntas número 5, 6 e 7 do formulário questionaram especificamente sobre a avaliação dos projetos de Unidades de Pronto Atendimento (UPA) e 
Unidades Básicas de Saúde (UBS). Ocorre que, desde 2011, o Ministério da Saúde elegeu alguns programas como prioritários para ampliação do acesso à saúde da população, dentre eles a ampliação da Rede de Atenção Básica, a reestruturação da Rede de Urgência e Emergência e o Plano Nacional de Doenças Crônicas não Transmissíveis. Em todos esses programas, existe a necessidade de construção, reforma ou ampliação de EAS, que passam necessariamente pela avaliação de projetos arquitetônicos nas Vigilâncias Sanitárias.

Nesse sentido, a GRECS/Anvisa tem utilizado os dados do levantamento realizado para planejar suas ações de coordenação do SNVS. Em relação à reestruturação da Rede de Urgência e Emergência foi feita a comparação entre o número de profissionais em cada estado com a demanda projetada pelo Ministério da Saúde, a partir da comunicação em portarias do investimento para a construção ou reforma de UPAS (Unidades de Pronto Atendimento) espalhadas por todo território nacional. Os quadros abaixo apresentam a relação do número de profissionais habilitados a analisar o projeto físico e o número de novos projetos a serem implantados, por região.

Tabela 1. Número de servidores públicos, de contratos precários, força total de trabalho, projetos de construção de UPA, projetos de reforma de UPA, total de projetos de UPA e relação de projetos de UPA por profissionais disponíveis. Região Nordeste.

\begin{tabular}{l|cccccc|c}
\hline \multicolumn{1}{|c|}{ Estado } & $\begin{array}{c}\text { Servidores } \\
\text { Públicos }\end{array}$ & $\begin{array}{c}\text { Contratos } \\
\text { Precários }\end{array}$ & $\begin{array}{c}\text { Força } \\
\text { de } \\
\text { trabalho } \\
\text { total }\end{array}$ & $\begin{array}{c}\text { Projetos de } \\
\text { construção } \\
\text { de UPA }\end{array}$ & $\begin{array}{c}\text { Projetos } \\
\text { de } \\
\text { reforma } \\
\text { de UPA }\end{array}$ & $\begin{array}{c}\text { Total } \\
\text { de } \\
\text { UPAS }\end{array}$ & $\begin{array}{c}\text { Relação } \\
\text { projetos } \\
\text { UPAS: } \\
\text { profissional }\end{array}$ \\
\hline Alagoas & 1 & - & 1 & 2 & 1 & 3 & $3: 1$ \\
Bahia & - & 1 & 1 & 13 & - & 13 & $13: 1$ \\
Ceará & - & 1 & 1 & 15 & 1 & 16 & $16: 1$ \\
Maranhão & - & - & - & 6 & - & 6 & $6: 1$ \\
Paraíba & 1 & - & 1 & 4 & - & 4 & $3: 1$ \\
Pernambuco* & 12 & - & 12 & 8 & - & 8 & $8: 12$ \\
Piauí & 2 & - & 2 & 2 & 2 & 4 & $2: 1$ \\
Rio G. Norte & 4 & 1 & 5 & 2 & - & 2 & $2: 5$ \\
Sergipe & - & - & - & - & 1 & 1 & $1: 1$ \\
\hline
\end{tabular}

- O estado de Pernambuco respondeu a enquete por telefone 
Tabela 2. Número de servidores públicos, de contratos precários, força total de trabalho, projetos de construção de UPA, projetos de reforma de UPA, total de projetos de UPA e relação de projetos de UPA por profissionais disponíveis.Região Norte

\begin{tabular}{l|cccccc|c}
\hline \multicolumn{1}{|c}{ Estado } & $\begin{array}{c}\text { Servidores } \\
\text { Públicos }\end{array}$ & $\begin{array}{c}\text { Contratos } \\
\text { Precários }\end{array}$ & $\begin{array}{c}\text { Total da } \\
\text { Força de } \\
\text { trabalho }\end{array}$ & $\begin{array}{c}\text { Projetos de } \\
\text { construção } \\
\text { de UPA }\end{array}$ & $\begin{array}{c}\text { Projetos } \\
\text { de } \\
\text { reforma } \\
\text { de UPA }\end{array}$ & $\begin{array}{c}\text { Total } \\
\text { de } \\
\text { UPAS }\end{array}$ & $\begin{array}{c}\text { Relação } \\
\text { projetos } \\
\text { UPAS: } \\
\text { profissional }\end{array}$ \\
\hline Acre & 3 & 3 & 6 & - & - & - & - \\
Amapá & - & - & - & - & - & - & - \\
Amazonas & 3 & 3 & 6 & - & - & - & - \\
Pará & - & - & - & 6 & - & 6 & 6 \\
Rondônia & 5 & - & 5 & - & - & - & - \\
Roraima & 1 & - & 1 & - & - & - & - \\
Tocantins & 1 & 1 & 2 & - & - & - & - \\
\hline
\end{tabular}

Tabela 3. Número de servidores públicos, de contratos precários, força total de trabalho, projetos de construção de UPA, projetos de reforma de UPA, total de projetos de UPA e relação de projetos de UPA por profissionais disponíveis.Região Centro-Oeste.

\begin{tabular}{l|cccccc|c}
\hline \multicolumn{1}{c|}{ Estado } & $\begin{array}{c}\text { Servidores } \\
\text { Públicos }\end{array}$ & $\begin{array}{c}\text { Contratos } \\
\text { Precários }\end{array}$ & $\begin{array}{c}\text { Total da } \\
\text { força de } \\
\text { trabalho }\end{array}$ & $\begin{array}{c}\text { Projetos de } \\
\text { construção } \\
\text { de UPA }\end{array}$ & $\begin{array}{c}\text { Projetos de } \\
\text { reforma de } \\
\text { UPA }\end{array}$ & $\begin{array}{c}\text { Total } \\
\text { de } \\
\text { UPAS }\end{array}$ & $\begin{array}{c}\text { Relação } \\
\text { projetos } \\
\text { UPAS: } \\
\text { profissional }\end{array}$ \\
\hline $\begin{array}{l}\text { Distrito } \\
\text { Federal }\end{array}$ & 2 & - & 2 & 1 & - & 1 & 1 \\
Goiás & 1 & - & 1 & 4 & 6 & 10 & $10: 1$ \\
Mato Grosso & 2 & 1 & 3 & 6 & 1 & 7 & $2 ; 1$ \\
$\begin{array}{l}\text { Mato Grosso } \\
\text { do Sul }\end{array}$ & 1 & - & 1 & - & - & - & - \\
\hline
\end{tabular}


Tabela 4. Número de servidores públicos, de contratos precários, força total de trabalho, projetos de construção de UPA, projetos de reforma de UPA, total de projetos de UPA e relação de projetos de UPA por profissionais disponíveis. Região Sudeste.

\begin{tabular}{l|cccccc|c}
\hline \multicolumn{1}{c|}{ Estado } & $\begin{array}{c}\text { Servidores } \\
\text { Públicos }\end{array}$ & $\begin{array}{c}\text { Contratos } \\
\text { Precários }\end{array}$ & $\begin{array}{c}\text { Total da } \\
\text { Força de } \\
\text { trabalho }\end{array}$ & $\begin{array}{c}\text { Projetos de } \\
\text { construção } \\
\text { de UPA }\end{array}$ & $\begin{array}{c}\text { Proposta } \\
\text { de } \\
\text { reforma } \\
\text { de UPA }\end{array}$ & $\begin{array}{c}\text { Total } \\
\text { de } \\
\text { UPAS }\end{array}$ & $\begin{array}{c}\text { Relação } \\
\text { projetos } \\
\text { UPAS: } \\
\text { profissional }\end{array}$ \\
\hline $\begin{array}{l}\text { Espírito } \\
\text { Santo }\end{array}$ & 1 & 1 & 2 & 1 & 2 & 3 & $1: 1$ \\
$\begin{array}{l}\text { Minas } \\
\text { Gerais }\end{array}$ & 15 & 3 & 18 & 8 & 4 & 12 & $12: 18$ \\
$\begin{array}{l}\text { Rio de } \\
\text { Janeiro }\end{array}$ & 8 & - & 8 & 9 & - & 9 & $1: 1$ \\
São Paulo & 65 & 8 & 73 & 21 & 6 & 27 & $27: 73$ \\
\hline
\end{tabular}

Tabela 5. Número de servidores públicos, de contratos precários, força total de trabalho, projetos de construção de UPA, projetos de reforma de UPA, total de projetos de UPA e relação de projetos de UPA por profissionais disponíveis. Região Sul.

\begin{tabular}{l|ccccccc|c}
\hline \multicolumn{1}{|c}{ Estado } & $\begin{array}{c}\text { Servidores } \\
\text { Públicos }\end{array}$ & $\begin{array}{c}\text { Contratos } \\
\text { Precários }\end{array}$ & $\begin{array}{c}\text { Total da } \\
\text { força de } \\
\text { trabalho }\end{array}$ & $\begin{array}{c}\text { Projetos de } \\
\text { construção } \\
\text { de UPA }\end{array}$ & $\begin{array}{c}\text { Projetos } \\
\text { de } \\
\text { reforma } \\
\text { de UPA }\end{array}$ & $\begin{array}{c}\text { Total } \\
\text { de } \\
\text { UPAS }\end{array}$ & $\begin{array}{c}\text { Relação } \\
\text { projetos } \\
\text { UPAS:profiss } \\
\text { ional }\end{array}$ \\
\hline Paraná & 13 & - & 13 & 4 & 1 & 5 & $5: 13$ \\
Rio G. Sul & 16 & - & 16 & 8 & - & 8 & $8: 16$ \\
S.Catarina & 4 & - & 4 & 8 & - & 8 & $2: 1$ \\
\hline
\end{tabular}

A comparação da força de trabalho $x$ a demanda proveniente das novas UPAS apresenta a constatação de que em alguns estados da região Norte, Nordeste e Centro-Oeste há sobrecarga para os profissionais responsáveis pela análise dos projetos de infraestrutura. Ainda soma-se o fato de que as Vigilâncias Sanitárias são responsáveis pela análise de outros equipamentos de saúde, como hospitais, clínicas, hemocentros além de edificações que não se identificam diretamente com a 
saúde, mas que também fazem parte do escopo do trabalho das Visas, como é o caso dos supermercados, creches, Institutos de longa permanência entre outros.

Observa-se ainda pela análise de dados que em alguns estados do Nordeste não há servidor público responsável pela análise dos projetos, cabendo esta atividade a profissionais contratados, o que fragiliza a instituição pública.

Ainda como consequência da relação feita entre os dados do levantamento da capacidade de avaliação de projetos arquitetônicos das VISAS e as demandas oriundas de programas do Ministério da Saúde, no âmbito do Plano Nacional de Doenças Crônicas não Transmissíveis, está prevista a ampliação dos serviços de radioterapia no País. Para tanto serão instalados aproximadamente 100 aceleradores lineares em diversos serviços distribuídos no País, que vão requerer reformas ou construções das estruturas físicas.

Em vista dessa demanda, a GRECS/Anvisa planejou um curso de capacitação para arquitetos e engenheiros das Vigilâncias Sanitárias estaduais e de capitais no tema. O curso ocorreu nos dias 20 e 21 de junho de 2013 na cidade de Brasília. Com o objetivo de apresentar as características destas edificações a fim de que quando os projetos forem encaminhados aos Estados/Capitais possam ser avaliados com mais celeridade e facilidade.

Dessa forma a GRECS/Anvisa, conhecedora da capacidade de avaliação de projetos arquitetônicos no País, tem planejado suas ações e coordenado o SNVS para melhor atender as demandas das políticas públicas de saúde brasileiras.

\section{Referências}

NADER, Rosa Maria. A avaliação como ferramenta para uma gestão pública orientada para resultados. O caso do Governo Federal Brasileiro. Santiago: X Congresso Internacional del CLAD sobre la Reforma del Estado y de la Administración Publica, CLAD, 2005.

PEREIRA, Luiz Carlos Bresser. Da Administração Pública a Gerencial. Revista do Serviço Público, 47(1):7-40, jan./abr., 1996.

PORTARIA $\mathrm{n}^{\circ}$ 1.344, de 29 de junho de 2012. Habilita Unidade de Pronto Atendimento (UPA 24h).

PORTARIA n ${ }^{\circ}$ 1.397, de 4 de julho de 2012. Habilita Unidade de Pronto Atendimento - UPA 24 horas. 
Anais dos III Congresso Iberoamericano de Direito Sanitário / II Congresso Brasileiro de Direito Sanitário

PORTARIA n 1.406, de 5 de julho de 2012. Habilita Unidade de Pronto Atendimento (UPA 24h).

PORTARIA n 931, de 10 demaiode 2012. Institui o Plano de Expansão da Radioterapia no Sistema Único de Saúde (SUS).

RDC/Anvisa $n^{\circ} 51$ de 06 de outubro de 2011. Diário Oficial da União, n 194, de 07 de outubro de 2011. 